\title{
Infectious Otitis Media
}

National Cancer Institute

\section{Source}

National Cancer Institute. Infectious Otitis Media. NCI Thesaurus. Code C84354.

Inflammation of the anatomical structures of the middle ear secondary to an infectious process. Bacterial etiology is most common, but both viral and fungal pathogens are also possible. Symptoms include erythema and edema of the tympanic membrane, pain, and possibly fever. In severe infections, inflammation and edema of the structures of the middle ear can lead to perforation of the tympanic membrane secondary to the buildup of pressure in the narrow space. 